8th Internationa1. Symposium on Electron Beam Ion Sources and Traps and their Applications, BNL, Upton, NY, November $6-8,2000$

BNL -68990

\title{
INVESTIGATION OF A PLASMA MODE IN EBTS
}

\author{
Ady Hershcovitch
}

CAD Department, Brookhaven National Laboratory, Upton New York 11766, USA

\begin{abstract}
A plasma related mode has been identified when EBTS operated with long trap length. The mode frequency scaling showed monotonic increased with confinement time. Initial scaling qualitatively suggested the mode to an electron beam driven ion cyclotron instability. However, a more quantitative evaluation is indicative of a drift mode. Nevertheless, the possibility of a structure mode, though unlikely, can not be completely excluded. The process of proper instability identification and stabilization is described.
\end{abstract}

\section{INTRODUCTION}

EBIS stability had been treated in a number of controversial papers [1,2]. This controversy extended to both experimental and theoretical papers. In the theoretical papers, plasma stability theory was applied in a rather cavalier way to EBIS devices. One of the reasons for the controversy is the fact that, in many of the papers on this subject matter, plasma theories have been applied to analyze experimental devices without first exploring whether those theories are valid for the experimental parameters they analyze. Kinetic theory, macro-, and micro-instabilities have been no exception. Additionally, in a number of these papers no distinction is made with regards to the class of instabilities (including those resulting from interactions with the EBIS structure).

Poor performance in some EBIS devices, especially when coupled with observation of rf signal, was occasionally blamed on plasma instabilities. In most of these experiments, identification of an instability was based solely on frequency detection by a frequency analyzer. If the value of the observed frequency matched the frequency a known plasma mode, positive instability identification was made. Gloom and doom predictions followed.

When trying to attribute anomalous behavior in an EBIS to various plasma instabilities, questions must be raised as to whether there is a simpler explanation [3] like misalignment. Or, is the generated if noise due to excitation and/or amplification of ,instabilities. Special care must be taken. when observations are based solely on spectrum analyzers.

In this paper investigation of an observed plasma mode is described. Although a specific mode is identified and analyzed, the work should be viewed as a broad, 
instabilities. Special care must be taken when observations are based solely on spectrum analyzers.

In this paper investigation of an observed plasma mode is described. Although a specific mode is identified and analyzed, the work should be viewed as a broad, general, approach to properly identifying and, if needed, to eliminate plasma instabilities in EBIS devices.

\section{Experimental Setup}

Measurements were performed on the Electron Beam Test Stand (EBTS), which is shown below in figure 1. Detailed description of EBTS can be found elsewhere [4].

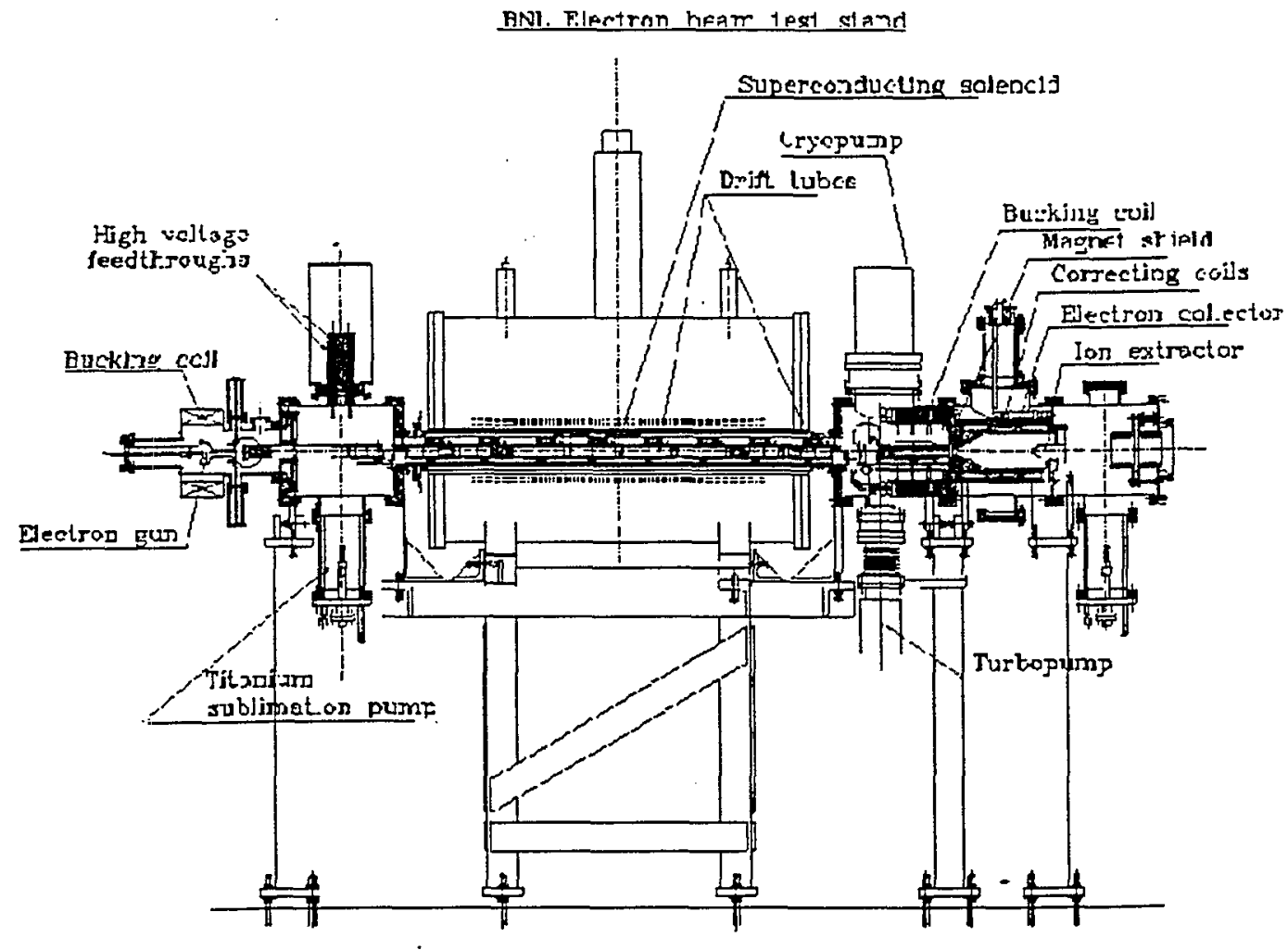

Figure 1. Layout of the experimental apparatus.

Two wire antennas are used for mode detection. In choosing an antenna, one mușt weigh antenna pick-up efficiency versus the explored spectra. Decision to use wire antennas was based on the need to explore as many modes as possible. One antenna is located in the chamber housing the titanium sublimation pump, while the other antenna is located in the chamber housing the turbomolecular pump. The signal from either antenna is amplified by a wide-band amplifier (MCL Model \# ZHL-1042J) with a bandwidth of $0-2.5 \mathrm{GHz}$. From the amplifier the signal is sent into a signal analyzer (Tektronix 2754P). 


\section{Results}

Frequencies observed with the spectrum analyzer are plotted in figure 2 as a function of the confinement. All measurements were performed with no external ion or gas injection. Basically, the trap contained residual gas ions: oxygen, hydrogen, and carbon ions. These frequencies were observed only with extended trap length of about $110 \mathrm{~cm}$. When the trap length was reduced to its "normal" length of $74 \mathrm{~cm}$, these frequencies could not be monitored. Other parameters were as follows: an electron beam current $2.2 \mathrm{~A}$, central magnetic field is 4.6 tesla, electron beam diameter at that magnetic field is $1.5 \mathrm{~mm}$, and electron beam energy in the trap of $15 \mathrm{keV}$.

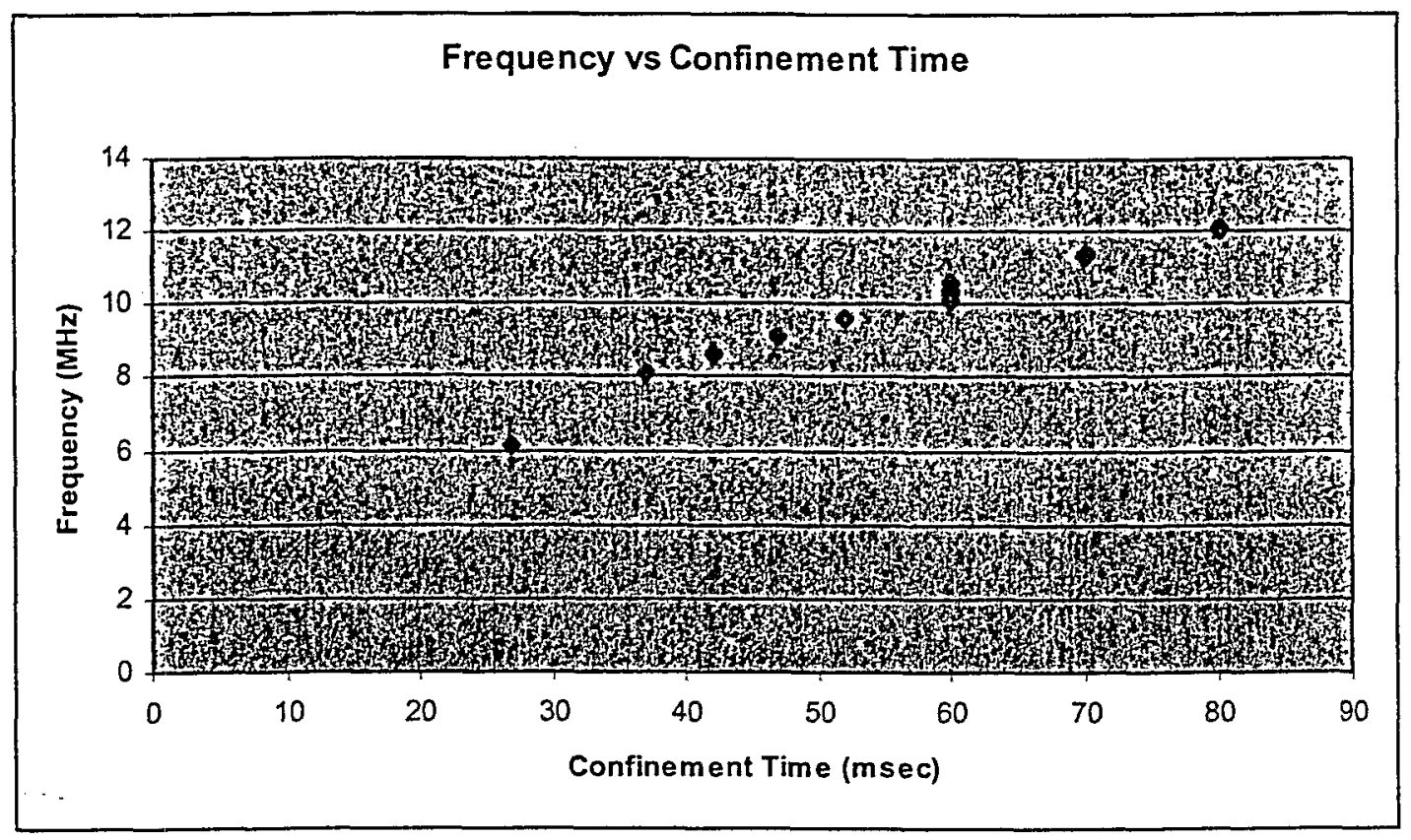

Figure 2. Observed frequencies versus the trap confinement period.

The observed frequencies were definitely plasma related. None of the modes could be observed with the electron beam off, or with the signal analyzer trigger outside the confinement time, i.e., triggering the signal analyzer before, or at the end of the confinement time. They could not be observed when either the electron beam was off, or when no ion trapping occurred.

As it can be seen from figure 2, the observed frequencies monotonically increase with confinement time. As a function of confinement time, the total ionic charge in the trap peaks at $60 \mathrm{msec}$ after monotonically increasing with trapping time. Peak ion charge was $16.4 \mathrm{nC}$. For confinement times longer than $60 \mathrm{msec}$, the number of ion charges decreases monotonically. Typically, in this mode of operation with residual gas, composition of the trapped ions was about $30 \%$ hydrogen, and the rest was divided between oxygen and carbon at a ratio of about $65 \%$ to $35 \%$ respectively [5]. Most probable charge states are $\mathrm{C}^{+3}-\mathrm{C}^{+4}$ and $\mathrm{O}^{+4}-\mathrm{O}^{+5}[5]$; those charge states are reached during the first $20 \mathrm{msec}$ of the confinement period. Maximal ion temperature is $200 \mathrm{eV}$ (about $10 \%$ of the potential well). 
A striking feature of the observed frequencies is the sharpness of the spectrum. Each mode has width of well below $1 \mathrm{MHz}$. The trace on the spectrum analyzer was smooth without the "fuzz" which is characteristic of plasma modes when displayed on an oscilloscope. Another rather unusual behavior is the fact that the frequencies detected at shorter confinement times were not observed at longer confinement times.

A possible reason for this seemingly strange behavior is due to limitation of pulsed if signals by spectrum analyzers as stated in the Tektronix 2754P manual. Other reasons could be selectivity by the automatic stabilization section of the YTO tuning oscillator [6]. Additional possible limitations may be due to mixer by products or resolution bandwidth.

\section{Analysis}

Electron beam generated conventional structure modes and/or excitations "ringing" driven by the variety of pulsed voltages can be excluded for the following reasons:

1. Structure modes require that the electron beam current exceed a value that is proportional to the electron beam energy. A good example is the well-known backward wave oscillator instability [6]. For this mode to occur the electron beam current I must satisfy the following condition I $>4 E / Z *(A / N)^{3}$, where $E$ is the electron beam energy, $Z$ is the circuit impedance, $N$ is the length of the structure in wavelengths, and $\mathrm{A}$ is a dimensionless geometrical parameter. Normal practice, by EBIS operators, to circumvent this instability is to raise the electron beam energy. In our case, the modes disappear when the voltage on the trap, and hence, the electron beam energy is reduced, i.e., opposite to conventional structure mode scaling.

2. Frequencies associated with square-wave pulsed voltage excitations in a fixed structure should be independent of the pulse length [8], or at most, inversely proportional to the pulse length. Here again, the opposite is true experimentally. Additionally, the modes can not be observed when voltages are pulsed, but the electron beam was off, and/or ion trapping did not occur.

Basically, the scaling of the observed modes is inconsistent with conventional structure modes or with pulsed voltage oscillations.

Having excluded conventional structure modes, plasma modes must be considered. Monotonic frequency increase with confinement time implies that the frequency of the mode must depend on a parameter, which increases monotonically with time. Therefore, most plasma instabilities can be excluded, since their dependence on the plasma frequency is inconsistent with the drop in ion charges for confinement times longer than $60 \mathrm{msec}$. Two parameters, which increase monotonically with time, are ion charge state $q$ and ion temperature.

It had been shown [9] that longitudinal current may excite ion-cyclotron oscillations. The derivation was done for an infinite homogenous plasma. That instability has a rather convoluted dispersion relation with two oscillation branches: at about the cyclotron frequency $\omega \approx n \Omega(1+A)$, where $\Omega$ is the ion cyclotron frequency, and $\mathrm{A}$ is a complicated term that is usually much smaller than 1 . The other branch is at $\omega / \xi$ where $\xi$ is larger than 1 . The observed frequencies range from 6.1 to $12.1 \mathrm{MHz}$. 
The cyclotron frequency for $\mathrm{C}^{+3}$ and $\mathrm{O}^{+4}$, e.g., is $17.48 \mathrm{MHz}$ in the center of the trap and $7.6 \mathrm{MHz}$ at the trap edge, where the magnetic field is about 2 tesla. Numerically, the mode is consistent for all confinement times with the second branch, however, it is basically temperature independent. If one extrapolate (not clear that it is justified) the dispersion relation to include density gradients, one could obtained a dependence of $\omega$ on temperature that may be consistent with observations.

Drift waves (a.k.a. universal modes) may be a better explanation for the observed phenomena. These modes occur in all magnetized plasmas that have transverse density gradients due to diamagnetic drift. The drift frequency $\omega_{*}$ is given by equation 1

$$
\omega_{*}=\mathrm{k}_{\perp} \mathrm{V}^{2} / 2 \Omega * \mathrm{~L}
$$

where, $V$ is the ion thermal velocity and $L=(1 / n) *(\mathrm{dn} / \mathrm{dr})$ is the density scale length, which in our case is roughly the plasma radius (also equal to $1 / \mathrm{k}_{\perp}$ ). For $200 \mathrm{eV}$ (upper limit temperature) $\mathrm{O}^{+4}$ in the center of the trap $\omega_{*}=15.7 \mathrm{MHz}$. Lower temperatures and/or carbon ions can easily account for the frequencies as low as $1 \mathrm{MHz}$, i.e., the range of observed frequencies is well accounted for by the plasma parameters. Therefore, there is an excellent numerical agreement between the observed frequencies and the existence of drift waves. Scaling wise, that agreement extends to the $\omega$. dependence on the confinement time, since in absence of cooling, ion heating rate is constant.

Another mode, which would give an equally good agreement is the current driven ion acoustic instability. Its frequency is given by equation 2

$$
\omega=\mathrm{k}^{2} \mathrm{C}_{\mathrm{s}} /\left[1+\left(\mathrm{k} \lambda_{\mathrm{D}}\right)^{2}\right]
$$

where, $C_{s}$ is the ion sound velocity, which is proportional to $\left(T_{e}\right)^{0.5}$, and $\lambda_{D}$ is the Debye length. At $200 \mathrm{eV}$, frequencies of up to $30 \mathrm{MHz}$ can be accounted for. However, the scaling is not as favorable as it is for the drift mode.

\section{Discussion}

Most existing theories of the plasma modes are based on derivations that assume quasi-neutral infinite plasmas. Additionally, those derivations lack the specifics of EBIS geometry. Adequate derivations [2,3] are yet to be applied to specific modes. The mathematics involved would be extremely lengthy. And, without detailed knowledge of the ion temperature and its time evolution, it would be an exercise in futility in this case. While derivations for ion cyclotron and ion acoustic instabilities do not account for the EBIS geometry, drift mode derivations have some level of consistency, since density gradients are included.

All plasma modes that have been considered are convective modes, and their derivations include long wavelength (along the magnetic field) approximations. These facts are consistent with the fact that the modes can be observed only in a long trap.

Structure mode derivations do not include ions (let alone trapped ions) in their dispersion relations. Thus, existing theories for structure modes are inconsistent with EBIS plasmas. Hence, structure modes can not be completely excluded. 
For both the ion cyclotron instability and ion acoustic instability to occur, the plasma frequency has to exceed the cyclotron frequency, or at least their ratio must exceed a certain parameter ratio. Therefore, should any of these instabilities occur, higher magnetic field could be used to stabilize them. And, ion cooling would stabilize the ion cyclotron instability.

From the limited data, it is impossible to determine whether the observed drift modes are unstable. Unstable drift modes are driven' by strong finite Larmor radius effect. Therefore, should unstable drift mode be encountered, ion cooling could reduce their growth rate.

\section{Conclusion}

Plasma modes have been studied in EBTS. Data supports the existence of universal modes in EBTS. It is not clear, at this junction, whether the observable is a drift wave instability, or just thermal drift wave oscillations. Further investigation is needed for positive determination.

EBTS and future BNL EBIS devices utilize, broader, larger diameter beams than early EBIS/EBIT devices. Since the Debye length is smaller than the beam diameter, plasma theory is applicable. To better identify, understand and stabilize EBIS instabilities, further theoretical and experimental work is needed.

As this and previous [11,12] work have shown, spectrum analyzers are not adequate for identification of instabilities in EBIS devices (unless the pulse length are very long like in EBIT). Tunable band pass filters should be used to study plasma modes.

\section{Acknowledgement}

This work was supported by the United States Department of Energy under contract DE-AC02$98 \mathrm{CH} 10886$. Fruitful discussions with Bob Schoepfer, Sasha Pikin and Mike Brennan are gratefully acknowledged.

\section{References}

1. See for example, panel discussion, N. Rostoker, moderator, in "Proceedings of International Symposium on Electron Beam Ion Sources and Their Applications", A. Hershcovitch, Editor, American Institute of Physics Conference Proceedings No. 188, American Institute of Physics, New York, 1989, pp. $179-187$.

2. A. Hershcovitch, Physica Scripta, T71, 34 (1996).

3. A. Hershcovitch, Rev. Sci. Instrum. 69, 668 (1998).

4. A. Pikin et al. in these proceedings.

5. A. Pikin, private communication 2000 .

6. Appendix B, Theory of Operation, HP Model $8569 \mathrm{~B}$ spectrum analyzer manual.

7. L.R. Walker, J. Appl. Phys. 24, 853 (1953).

8. J.M. Brennan, private communication 2000.

9. W. Drummond and M. Rosenbluth, Phys. Fluids, 5, 1507 (1962).

10. See for example, R.E. Marrs, NIM B 149, 182 (1999).

11. A. Hershcovitch, and P. Politzer, Phys. Fluids, 36, 1365 (1976).

12. A. Hershcovitch, and P. Politzer, Phys. Fluids, 22, 249 (1979). 